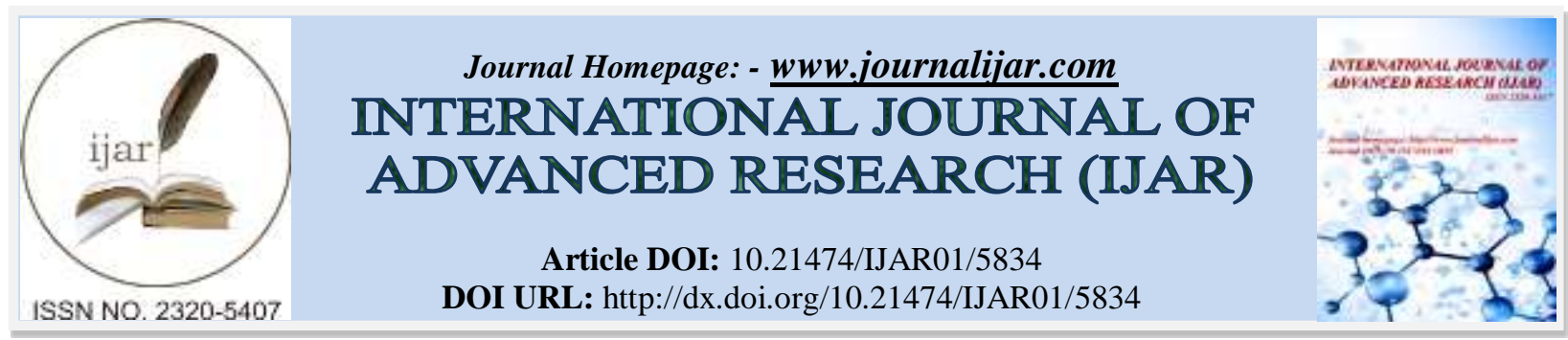

RESEARCH ARTICLE

\title{
BIOLOGICAL IMPACT OF METFORMIN VERSUS THIAZOLIDINEDIONE ON ALVEOLAR BONE OF INDUCED DIABETIC RATS (A HISTOLOGICAL AND IMMUNO-HISTOMORPHOMETRIC STUDY).
}

Marwa A. M. El-Shiekh ${ }^{1}$ and Heba A. Adawy ${ }^{2}$.

1. Lecturer of Oral and Dental Biology, Faculty of Dental Medicine for girls, Al-Azhar University, Cairo-Egypt.

2. Assistant Prof. of Oral and Dental Biology, Faculty of Dental Medicine for girls, Al-Azhar University, CairoEgypt.

\section{Manuscript Info}

Manuscript History

Received: 11 September 2017

Final Accepted: 13 October 2017

Published: November 2017

Key words:-

Diabetes, anti-diabetic drugs, Metformin,

Thiazolidinedione, alveolar bone, Osteoprotogrin.

\section{Abstract}

Objective: To evaluate how the most currently used anti-diabetic drugs (pioglitazone \& metformin) can secondarily affect diabetic alveolar bone.

Materials and methods: 70 adult male rats (170-200 g) were obtained. Diabetes was induced in 60 animals by intraperitoneal administration of nicotinamide (NA) $(270 \mathrm{mg} / \mathrm{kg}) 15 \mathrm{~min}$ before IV administration of STZ $(65 \mathrm{mg} / \mathrm{kg})$. The rats were divided into four groups: Control; Group (I): composed of 10 rats without diabetes. Experimental groups; Group (II) composed of 20 diabetic rats. Group (III): 20 diabetic rats received pioglitazone ( $4 \mathrm{mg} / \mathrm{kg} /$ day). Group (IV): composed of 20 diabetic rats received metformin $(250 \mathrm{mg} / \mathrm{kg} /$ day $)$. By the end of the experimental period ( 4 weeks), all rats were sacrificed. The mandibles specimens were processed for histological examination and immunostained by Osteoprotogrin for immunohistomorphometry.

Results: The histological results in (group IV) revealed minimal osteoporotic features of alveolar bone in relation to (group III) that showed an obvious deterioration of the alveolar bone. Immunohistochemical results showed mild to strong stained areas in group (IV) and negative immune-stain for all regions except for few areas appeared with very mild stain in group (III). Statistical analysis revealed that no significant difference between group I and group IV. Moreover, the difference between group IV and groups II and III was significant.

Conclusion: Our result stands as a source of data regarding the in vivo superior influence of anti-diabetic drug Metformin versus

Thiazolidinedione (Pioglitazone) on diabetic alveolar bone health by its inhibitory effect on diabetic bone loss.

Copy Right, IJAR, 2017,. All rights reserved. 


\section{Introduction:-}

Diabetes mellitus (DM) is a highly prevalent global disease associated with long-term micro-vascular and macrovascular complications. The prevalence of diabetes mellitus in general population is continuously increasing and it has been estimated that the number of diabetic patients will reach 592 million worldwide by the end of 2035. (Viviana et al., 2017)

Osteoporosis also is a major public concern. The association between diabetes and bone health has been a matter of debate for a long time. Skeletal affection in diabetes was first discussed 80 years ago. Now, considerable volume of data is available, that indicate that metabolic and endocrine alterations beside micro-angiopathy accompanying diabetes can negatively affect skeletal bone quantity and/or quality that can be named "diabetic osteopathy". (Adami, 2009)

"Diabetic osteopathy" includes osteopenia, osteoporosis and an increased incidence of low-stress fractures. A broad animal studies suggested that diabetic bone may be more fragile than non- diabetic. An increased risk of fracture has been associated with both diabetes Type I and Type 2 (Silvano, 2009). Osteoporosis, and related fractures, is a clinically significant and commonly underestimated problem in diabetes patients. (Leidig-Bruckner et al., 2014)

Patients with osteoporosis are almost under primary care of physicians for management of other medical conditions in addition to their osteoporosis. It seems that it is mandatory to be aware of how the prescribed medications may adversely affect the skeleton and increase the risk of fractures.

Two classes of oral hypoglycemic drugs are used for diabetes treatment to directly improve insulin action as their primary effect: Biguanides (metformin) and thiazolidinediones (rosiglitazone and pioglitazone). Thiazolidinediones have been introduced in the treatment of diabetes type 2 (T2DM) since the late 1990s. Both rosiglitazone and pioglitazone gained widespread use. However, in 2010, due to increased risk of cardiovascular events associated with its use, the European Medicines Agency recommended suspension of rosiglitazone use and the Food and Drug Administration (FDA) severely restricted its use. (Consoli \& Formoso, 2013)

Thus, pioglitazone is the only thiazolidinedione still significantly used for treating T2DM and it is the only molecule of this class still listed in the American Diabetes Association (ADA) and European Association for the Study of Diabetes (EASD) 2012 Position Statement. Meanwhile, according to the (ADA) and (EASD), metformin (in the absence of contraindications) is considered the first choice for oral treatment of T2DM. (Nathan, 2006 ; Inzucchi, 2015)

Thiazolidinediones (TZDs) are insulin-sensitizing drugs that are agonists of peroxisome proliferator-activated receptor gamma (PRARy), a nuclear transcription factor that is expressed in bone marrow stromal cells, osteoblasts and osteoclasts. They increase insulin sensitivity by acting on adipose, muscle, and liver to increase glucose utilization and decrease glucose production (Hauner, 2002). They are excellent therapeutic approach for treating T2DM, but their prolonged use promote some adverse effects, such as fluid retention and weight gain (Bosi, 2007). Clinical evidence suggests that these drugs can cause bone loss and increase fracture risk. (Gilbert, 2015)

Metformin, a widely accepted first line agent in diabetes treatment, stands out not only for its antihyperglycemic properties but also for its beneficial effects in improvement of endothelial dysfunction, hemostasis and oxidative stress, insulin resistance, lipid profiles, and fat redistribution. These properties have contributed to the decrease of adverse cardiovascular outcomes. There is increasing evidence from in vivo and in vitro studies supporting its antiproliferative role in cancer and possibly a neuroprotective effect. Metformin's negligible risk of hypoglycemia in monotherapy and few drug interactions of clinical relevance give this drug a high safety profile. (Rojas \& Gomes,2013)

Metformin increases insulin sensitivity in T2DM patients. It acts by decreasing hepatic glucose production and increasing glucose uptake in muscle. It likely improves glucose metabolism via the activation of AMP-activated protein kinase (AMPK), which results in the suppression of fatty acid synthesis, the stimulation of fatty acid oxidation in the liver, an increase in muscle glucose uptake. (Madsen, 2015) 
Osteoprotegerin (OPG) is a member of the tumor necrosis factor (TNF) receptor family which plays a key role in the physiological regulation of osteoclastic bone resorption. It is a glycoprotien, produced by osteoblasts and marrow stromal cells (Kostenuik \& Shalhoub, 2001). It is a soluble decoy receptor for receptor activator of nuclear factorkappaB ligand (RANKL), osteoclast differentiation factor, which inhibits both differentiation and function of osteoclasts. In vivo studies have demonstrated that OPG deletion results in enhanced remodeling of bone and osteoporosis, whereas OPG overexpression leads to osteopetrosis in mice. (Udagawa et al., 2000)

Several epidemiological studies have investigated the effects of antihyperglycaemic drugs on the fracture risk in diabetes and incident hip fractures. (Nicodemus \& Folsom, 2001 ; Vestergaard et al.,2005)

In this context it is important to evaluate how the most currently used anti-diabetic drugs mainly (pioglitazone \& metformin) can secondarily affect the health of diabetic alveolar bone.

\section{Materials and Methods:-}

\subsection{Animal selection:-}

70 adult male rats (170-200 g) were obtained from Animal House, Faculty of Medicine, Cairo University. The rats were kept under standard environmental conditions at room temperature $25 \pm 2{ }^{\circ} \mathrm{C}$, and $12 \mathrm{hrs} \mathrm{light/dark}$ cycle. Animals were fed with standard diet and tap water ad libitum.

\subsection{Induction of diabetes in rats:-}

Diabetes was induced in 60 overnight fasted animals by intraperitoneal administration of nicotinamide (NA) (270 $\mathrm{mg} / \mathrm{kg}) 15 \mathrm{~min}$ before IV administration of Streptozotocin (STZ) $(65 \mathrm{mg} / \mathrm{kg})$. STZ was dissolved in $0.1 \mathrm{M}$ citrate buffer $(\mathrm{pH} 4.5)$ and nicotinamide was dissolved in physiological saline (Kim et al.,2014). Peripheral blood was harvested from vena caudalis $72 \mathrm{hr}$ post-injection to assess blood glucose (BG) level, rats with BG more than 300 $\mathrm{mg} / \mathrm{dL}$ indicated the successful induction of diabetes and were used in this study.

\subsection{Groups design:-}

The rats were divided into four groups; Group I (control group); composed of 10 normal rats. Experimental groups; Group (II) (Diabetic group): composed of 20 diabetic rats that didn't receive any treatment. Group (III): 20 diabetic rats that received pioglitazone $(4 \mathrm{mg} / \mathrm{kg} /$ day $)$ suspended in distilled water in a dose volume of $5 \mathrm{ml} / \mathrm{kg}$ given orally by gastric tube (a dose equal to the maximum recommended human oral dose of $45 \mathrm{mg} /$ day) for 4 weeks (Hoda et al., 2009). Group (IV): composed of 20 diabetic rats that received metformin. The dose was calculated according to clinically relevant human dose. Metformin $(250 \mathrm{mg} / \mathrm{kg} /$ day $)$ dissolved in vehicle solution (5\% sodium carboxymethylcellulose) was administered by gavage for 4 weeks. (Okonkwo \& Okoye, 2014; Xue Han et al., 2017)

\subsection{Sample collection:-}

At the end of the experimental period ( 4 weeks), rats' scarification was done by slaughter. The heads were immediately dissected to obtain the mandibular molar area. The specimens were decalcified then processed and stained by routine haematoxylin \& eosin for examining bone micro-architecture and immunostained by Osteoprotogrin marker to evaluate the bone cellular activity by histomorphometry.

\subsection{Evaluation of OPG immunostaining by image analysis (immunohistomorphometry):-}

The number of positively reacting immunostaining of OPG was counted. Counting was based on the area with the highest percentage of positive cells; each stained cell was regarded as positive regardless of the staining intensity. The sites expressing a positive immune reaction with the OPG monoclonal antibody are identified by brown deposits of the chromogen. The stronger the immune reaction was the darker the chromogen intensity.

The study proposal was approved by the Research Ethics Committee (REC), Faculty of Dental Medicine for Girls, Al-Azhar University.

\section{Results:-}

\subsection{Histological results:-}

The control group (group I) showed normal alveolar bone structure; average normal thickness of bone trabeculae lined by osteoblasts and containing average normal number, size and arrangement of osteocytes. The bone trabeculae appeared with multiple resting and few reversal lines. Variable sized cellular bone marrow spaces were also seen (fig. 1A). 
The untreated diabetic group (group II) showed some structural alterations; where the bone trabeculae appeared thin with intervening wide fatty marrow spaces. The bone trabeculae lacked the osteoblast lining in many areas with minimal resting lines and multiple reversal lines, the osteocytes appeared few in number with areas seemed to lack their presence. The wide fatty bone marrow spaces showed multiple inflammatory cells. (fig. 1B)

Pioglitazone treatment group (group III) showed an obvious deterioration of the alveolar bone; where the trabeculae appeared very thin and loosing most of its osteoblastic lining. Osteocytes were irregularly arranged and showed eccentric nuclei with large sized osteocytic lacunae. Wide areas of intervening fibrous marrow spaces containing multiple inflammatory cells were seen between the trabeculae. (fig.1C)

Metformin treatment group (group IV) recorded minimal tissue alterations where thick bone trabeculae appeared with intervening average normal sized marrow spaces. The osteoblasts lined most of the bone trabeculae and the osteocytes showed also average normal number with irregular arrangement. (fig. 1D).

\subsection{Immunohistological results:-}

The control group (I): showed mild to strong immune- staining with OPG , where the stained areas were mostly seen in the peripheries of the bone trabeculae surrounding the marrow spaces (osteoblast linning) and also were scattered through the trabeculae ( some osteocytes)(fig.2A). Group (II): minimal to negative immune-staining was seen in this group, the stained areas were confined to few areas scattered through the bone trabeculae (few osteocytes). There was an absence of the immune-stain at the peripheries of the trabeculae (osteoblastic linnig).(fig.2B) Group (III): The specimens of this group showed negative immune-stain for all regions except for very mild stain that could be seen at few peripheral areas of the thin bone trabeculae.(fig 2 C). Group (IV): The bone trabeculae of this group showed mild to stong stained areas, most of the stained areas were scattered through trabeculae (osteocytes) and few were seen at their peripheries(osteoblasts)(fig2D)

\subsection{Immunohistomorphometrical results: (Image analysis and Statistical results):-}

Analysis of data was performed using SPSS 17 (Statistical Package for Scientific Studies) for Windows. The mean area percent of immunoexpression was expressed in the form of mean, standard deviation (SD). Data were explored for normality using Kolmogorov-Smirnov test of normality. The results of Kolmogorov-Smirnov test indicated that most of data were normally distributed (parametric data), therefore one-way analysis of variance (ANOVA) test was done. This was followed by Tukey's post hoc test since a significant difference was detected.

-Results were expressed in the form p-values that were differentiated into:

* Non-significant when p-value $>0.05$

* Significant when p-value $\leq 0.05$

The greatest mean value was recorded in group I, followed by group IV, then group II. The lowest value was recorded in group III. One way analysis of variance (ANOVA) test revealed that the difference between the control and experimental groups at 4 weeks was significantly different $(\mathrm{P}<0.0001)$. Tukey's post hoc test revealed no significant difference between group I and group IV. Moreover, the difference between group IV and groups II\&III was significant (Table 1) 


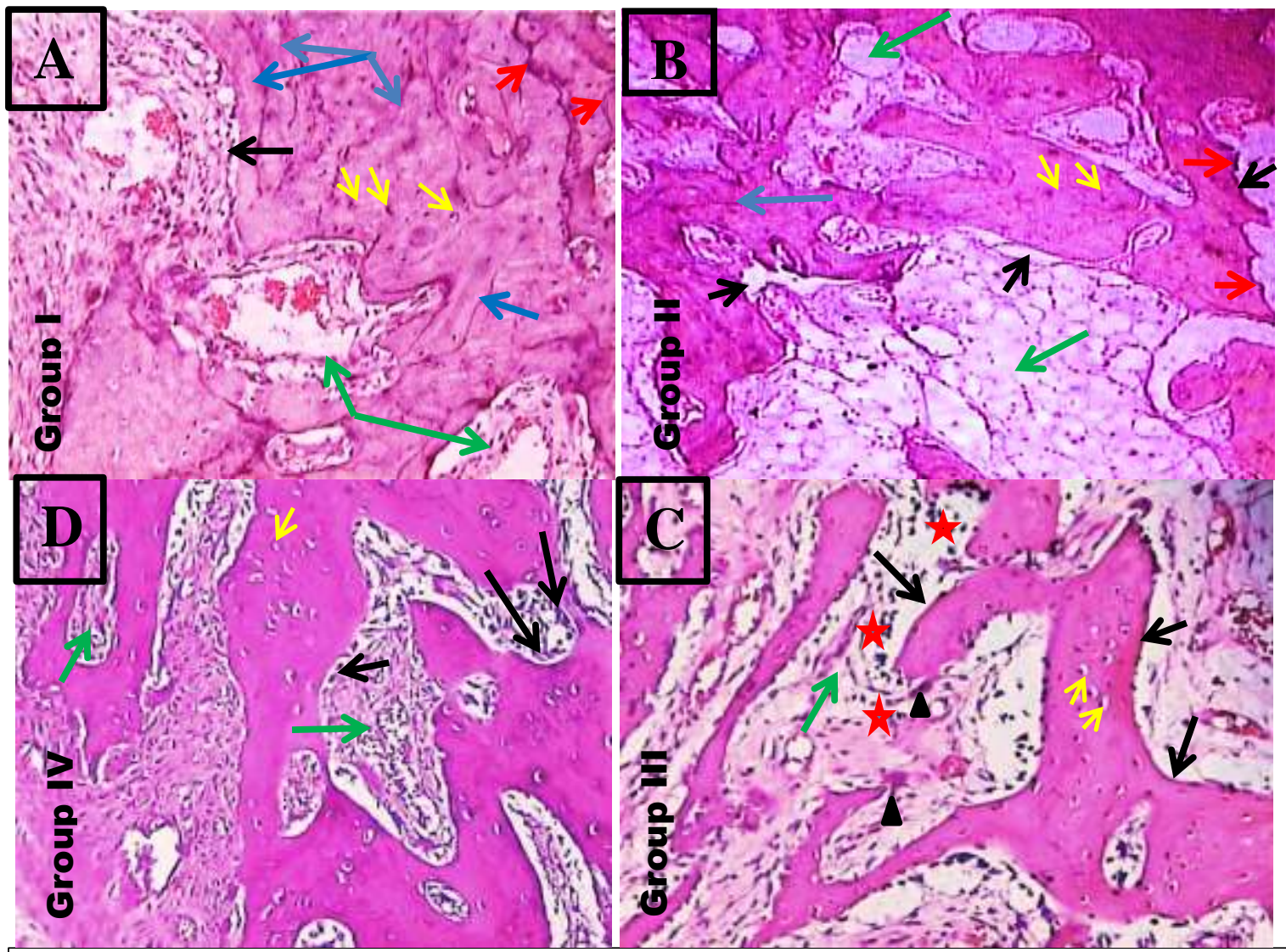

Fig (1):- Photomicrographs of Control group I (A), untreated diabetic group II (B), Pioglitazone treated group III (C) \& Metformin treated group IV (D).( Black arrows:osteoblast lining. Yellow arrows: osteocytes Red arrows: reversal lines. Green arrows: bone marrow spaces. Blue arrows: resting lines. Arsticks:

Inflammatory cells. Arrow heads: osteoclasts)

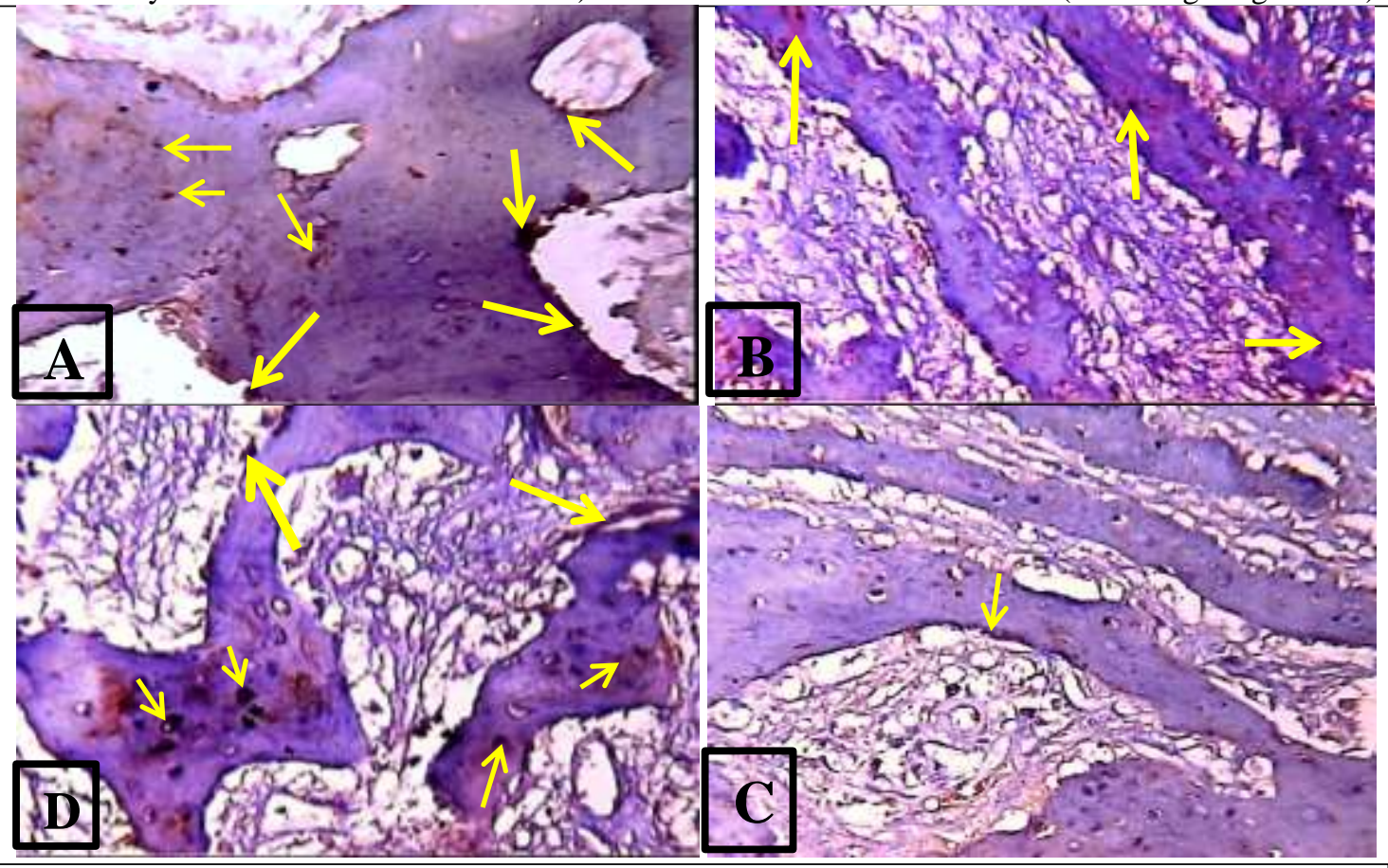

Fig (2):- Photomicrographs of all groups immune-stained with OPG

(OPG X100) 


\begin{tabular}{|l|l|l|l|l|}
\hline & Group I & Group II & Group III & Group IV \\
\hline Mean & $12.136^{\mathrm{c}}$ & $7.138^{\mathrm{b}}$ & $2.798^{\mathrm{d}}$ & $9.372^{\mathrm{a}, \mathrm{c}}$ \\
\hline Std Dev & 2.636 & 1.365 & 1.164 & 2.544 \\
\hline Max & 17.357 & 7.617 & 4.815 & 12.521 \\
\hline Min & 10.177 & 4.927 & 2.067 & 6.223 \\
\hline F value & 22.72 & \multicolumn{5}{|l|}{} \\
\hline P value & $<.0001^{*}$ & \multicolumn{4}{|l|}{} \\
\hline
\end{tabular}

Table (1):- Area percent of expression in all groups and significance of the difference using ANOVA test

Tukey's post hoc test: means with different superscript letters are significantly different. *significant at $\mathrm{p}<0.05$.

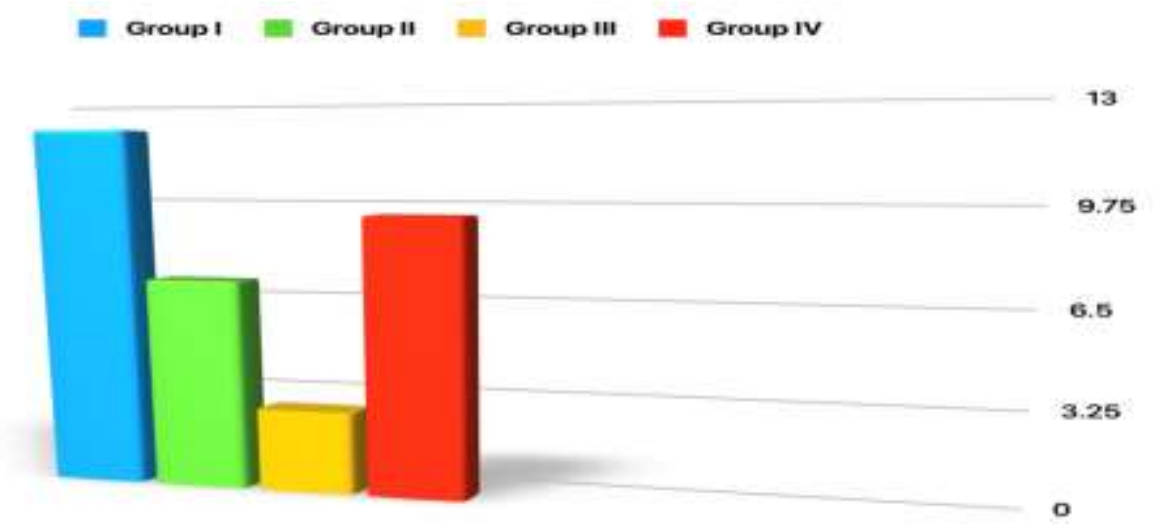

Chart representing percentage of immune expression areas in all groups.

\section{Discussion:-}

Bone health is an important consideration in diabetes. Diabetes complications and osteoporotic fractures are two of the most important causes of morbidity and mortality in older patients, and they share many features, including genetic susceptibility, molecular mechanisms, and environmental factors. The linkage between these two chronic diseases creates a possibility that certain anti-diabetic therapies may affect bone function. The treatment of Type 2 diabetes mellitus (T2DM) has been improved in the past two decades with the development of new therapeutic drugs (Vianna et al., 2017). The impact of diabetes and two of mostly used anti-diabetic medications on alveolar bone health was estimated in the present study.

The experimental model of STZ-NA-induced diabetes was used in this study as it has some similarities with T2DM in humans with reduced B-cell mass as well as metabolic defects in the insulin-secreting cells. Therefore, this diabetic model provides an advantageous tool for pharmacological investigations of new insulinotropic agents in humans. (Szkudelski et al., 2013)

It was demonstrated that administration of $60 \mathrm{mg}$ of STZ and $90 \mathrm{mg}$ of nicotiamide (NA) per $\mathrm{kg}$ body weight induced relatively mild diabetes after four to six weeks after diabetes induction. Furthermore, the used dosage of nicotiamide $270 \mathrm{mg} / \mathrm{kg}$, given intraperitoneally $15 \mathrm{~min}$ before STZ administration (65 mg/kg i.v.) yields animals with moderate and stable non-fasting hyperglycemia and $40 \%$ preservation of pancreatic insulin stores as it partially protects against STZ-induced $\beta$-cell necrosis. (Masillo et al., 1998; Szkudelski, 2012)

Bone quality is normally preserved by the process of bone remodeling; that comprises continuous resorption and formation of bone to substitute old tissue with new tissue. Equilibrium between osteoclast-dependent bone resorption and bone formation (which relies on osteoblast activity), is essential for the maintenance of bone mass (Schwartz, 2017). These features were clearly seen in the specimens of control group (I) of our study, through the presence of multiple resting lines; denoting bone formation and reversal lines; denoting bone resorption. (fig. 1 A).

Some of the proteins involved in the interaction between osteoblasts and osteoclasts have been identified. Receptor activator of nuclear factor-kappaB ligand (RANKL) is produced by osteoblasts and exerts its effects through binding to its receptor (RANK) on osteoclast precursor cells. Binding results in activation of osteoclasts. Osteoblasts also 
produce osteoprotegerin (OPG), a potent inhibitor of osteoclast formation and a decoy receptor for RANK. The relative ratio of OPG and RANKL in the bone may determine the number of active osteoclasts, bone resorption rate, and bone mass (Coetzee \& Kruger, 2004). So, OPG was used in the present study as marker for degree of bone resorption inhibition.

In the present study, the diabetic group (II) when compared to control group (I), showed osteoporotic features reflected histologically by reduction in trabecular bone thickness and loss of normal bone architecture (fig.1B). These findings were reported and explained by many studies; studies of bone density in patients with Type 1 diabetes mellitus (T1DM) and those with T2DM have been reported to have a higher incidence of fracture (Qing et al., 2007). Other studies stated that T2DM compromises bone microarchitecture by inducing abnormal bone cell function and matrix structure with increased osteoblast apoptosis, diminished osteoblast differentiation, and enhanced osteoclast-mediated bone resorption. These alterations result in attenuated remodeling process where circulating levels of biochemical markers of bone formation and resorption are decreased (Rubin, 2015). This was in commitment with our immunohistochemical and statistical findings; seen in the decreased OPG immunostainabilty of the specimens(fig.2B), and the lower mean value recorded in group II than group I with statistical significant decrease in OPG stainability.(table 1)

Hyperglycemia is a major contributing factor in the low bone turn over that occurs in diabetes; bone formation as well as bone resorption is inhibited by hyperglycemia through modulation of the osteoblast/osteoclast phenotype and function. It is speculated that low turnover of bone in diabetes lead to decreased bone quality (Dobnig et al., 2006). Furthermore, the Glucose-dependent insulinotropic peptide (GIP) receptors present on osteoblasts, osteocytes, and chondrocytes and are responsible for collagen type I expression and alkaline phosphatase activity, is known to be down regulated in DM because of the persistent elevations in (GIP). Thus, contribute to low bone quality and higher fracture rates (Zong et al., 2007). The low bone turnover, in this study, was confirmed in group (II) by the reduction in both resting and reversal lines.(Fig1B)

Thiazolidinedione (TZD) is one of the hypoglycemic drugs used for treatment of T2DM. Pioglitazone is the only thiazolidinedione still significantly used for treating T2DM. Several studies propose that the effects of TZDs on bone are a drug-class effect. (Lecka-Czernik et al., 2002; Nathan, 2006; Gilbert \& Pratley, 2015)

In the present study, Pioglitazone treated group (III) showed deterioration of the alveolar bone seen histologically as thin wiry bone trabeculae, with the loss of most of their osteoblastic lining. The trabeculae were widely separated by large fibrous marrow spaces. Increased lacunar size of osteocytes observed in (fig 1C) was similarly seen by Lane $e t$ al. (2006), who stated that the osteocyte-lacunar size would increase in glucocorticoid induced osteoporosis, their study observed changes in elastic modulus surrounding osteocyte lacunae, reduced mineral to matrix ratios in the same areas and an increase in lacunar size. This finding suggests that osteoporosis may alter the metabolism and function of the osteocyte and is not just inducing its death.

The explanation for the TZD-induced bone loss is demonstrated mainly by the mechanism of peroxisome proliferator-activated receptor-gamma (PPAR $\gamma$ ). TZDs increase insulin sensitivity through the activation of PPAR $\gamma$. Activation leads to the permanent conversion of osteoblastic cells to adipocytes and, additionally, suppresses the particular genetic expression of osteoblasts and their phenotype. (Lecka-Czernik et al., 2002)

The suppression in osteoblastic expression in our study was confirmed in pioglitazone treated group (III) by the OPG immunostaining and their statistical results, as the specimens appeared minimally or even negatively stained (fig 2C), and it showed the least mean value of OPG immunostainabilty among all the groups. This concept was supported by in vitro and in vivo studies that suggested that PPAR $\gamma 2$ is a positive regulator of adipocyte differentiation and acts as a dominant-negative regulator of osteoblast differentiation with greater support for osteoclastogenesis.(Ali et al., 2005)

Metformin is a widely used anti diabetic drug that improves glucose metabolism via the activation of AMP-activated protein kinase (AMPK), the exact effect of AMPK on bone metabolism is not yet understood. Some reports suggest that AMPK modulates bone cell differentiation and function. (McCarthy, 2016).

In the present study the positive effect of metformin on alveolar bone was clearly noticed and confirmed by the histological findings as the trabeculae restored their average normal thickness and continuation, with clear 
osteoblastic lining and the osteocytes appeared with proper arrangement and shape (fig 1D). Beside these findings our immunohistochemical and statistical results also supported the metformin osteogenic effect by the increase in the OPG stainability (fig. 2D), where group IV showed a statistically significant increase in the OPG stainability when compared with group II and group III.

Our findings were in accordance with studies that have reported direct osteogenic effect of anti-diabetic drug metformin on osteoblast-like cells in culture (McCarthy, 2016). Others provided an evidence that metformin been shown to enhance osteoblasts differentiation and inhibit osteoclast differentiation in vitro and has a direct inhibition effect on bone loss in OVX rats (Gao et al., 2010). But were against other studies that indicated that metformin has no effect on bone mass in vivo or fracture healing in rodents.(Jeyabalan et al., 2013)

The mechanisms through which metformin regulates bone metabolism are not fully known. Results from in vitro and animal studies have shown that metformin has a positive effect on osteoblast differentiation by activating the osteoblast-specific Runx2 (runt-related transcription factor 2) transcription factor via the AMPK/USF-1/SHP regulatory cascade. Metformin also has an adverse effect on osteoclast differentiation by decreasing the proosteoclastic cytokine receptor activator of nuclear factor $\mathrm{\kappa B}$ ligand (RANKL) and increasing osteoprotegerin levels. (Kasai et al., 2009; Mai et al., 2011)

Moreover, recent studies have indicated antioxidative properties of metformin. It exerts intracellular antioxidant properties by decreasing reactive oxygen species (ROS) production through the inhibition of protein kinase $\mathrm{C}$ activity (Marycz et al., 2016). Oxidative stress (OS) in the diabetic condition contributes to defective bone features. Antioxidative supplementation deals with excessive (ROS) may improve cellular metabolism and can be a valuable adjunctive strategy in treating diabetic bone disease, accelerating bone healing, and improving osteointegration. (Bacevic et al., 2017)

\section{Conclusion:-}

Beside its well-known anti hyperglycemic effect, Metformin showed a positive osteogenic effect on osteoporotic diabetic alveolar bone versus Thiazolidinedione (pioglitazone) that increased diabetic alveolar bone deterioration on the other side.

\section{Recommendations:-}

Anticipated beneficial effect of metformin on other diabetic oral tissues needs to be evaluated by future studies.

\section{References:-}

1. Adami S (2009) "Bone health in diabetes: considerations for clinical management". Current medical research and opinion. 25:1057-1072. doi: 10.1185/03007990902801147

2. Ali AA, Weinstein RS, Stewart SA, Parfitt AM, Manolagas SC, Jilka RL.(2005) "Rosiglitazone causes bone loss in mice by suppressing osteoblast differentiation and bone formation." Endocrinology. 146(3):1226-1235. doi:10.1210/en.2004-0735

3. Bacevic M, Brkovic B, Albert A, Rompen E, Radermecker RP, Lambert F (2017) "Does Oxidative Stress Play a Role in Altered Characteristics of Diabetic Bone? A Systematic Review." Calcif Tissue Int. 101(6):553563. doi: 10.1007/s00223-017-0327-7

4. Bosi E, Camisasca RP, Collober C, Rochotte E, Garber AJ. (2007)"Effects of vildagliptin on glucose control over 24 weeks in patients with type 2 diabetes inadequately controlled with metformin" Diabetes Care.30(4):890-895. doi:10.2337/dc06-1732

5. Coetzee M \& Kruger MC (2004) "Osteoprotegerin-receptor activator of nuclear factor-kappaB ligand ratio: a new approach to osteoporosis treatment?" South Med J.97(5):506-511.

6. Consoli A\& Formoso G. (2013) "Do thiazolidinediones still have a role in treatment of type 2 diabetes mellitus?"Diabetes Obes Metab. 15(11):967-977. doi: 10.1111/dom.12101

7. Dobnig H, Piswanger-solkner JC and Roth M. (2006) "Type 2 diabetes mellitus in nursing home patients: effects on bone turn over, bone mass, and fracture risk". J.clin. endocrinol.metab.91:3355-3363. doi:10.1210/jc.2006-0460

8. Gao Y, Li Y, Xue J, Jia Y, Hu J. (2010) "Effect of the anti-diabetic drug metformin on bone mass in ovariectomized rats." Eur J Pharmacol. 10;635(1-3):231-236. oi: 10.1016/j.ejphar.2010.02.051. 
9. Gilbert MP and Pratley RE. (2015) "The impact of diabetes and diabetes medications on bone health" Endocr Rev. Apr; 36(2):194-213. doi: 10.1210/er.2012-1042.

10. Gilbert MP, Pratley RE. (2015) "The impact of diabetes and diabetes medications on bone health." Endocr Rev. 36(2):194-213. doi:10.1210/er.2012-1042

11. Hauner H. (2002) "The mode of action of thiazolidinediones." Diabetes Metab Res Rev.18 (2):10-15.

12. Hoda W.El Gawly, Mona K.Tawfik, Maha F. Rashwan, Ali S. Baruzaig.(2009) "The effect of pioglitazone on the liver of streptozotocin-induced diabetic albino wistar rats. Eur Rev Med Pharmacol Sci. 13: 443-451

13. Inzucchi SE, Bergenstal RM, Buse JB, Diamant M, Ferrannini E, Nauck M, Peters AL, Tsapas A, Wender R, Matthews DR. (2015) "Management of hyperglycemia in type 2 diabetes, 2015: a patient-centered approach: update to a position statement of the American Diabetes Association and the European Association for the Study of Diabetes." Diabetes Care.38 (1):140-149.

14. Jeyabalan J, Viollet B, Smitham P, Ellis SA, Zaman G, Bardin C, Goodship A, Roux JP, Pierre M, Chenu C. (2013) "The anti-diabetic drug metformin does not affect bone mass in vivo or fracture healing." Osteoporos Int. 24(10):2659- 26570. doi: 10.1007/s00198-013-2371-0.

15. Kasai T, Bandow K, Suzuki H, Chiba N, Kakimoto K, Ohnishi T, Kawamoto S, Nagaoka E, Matsuguchi .T (2009) "Osteoblast differentiation is functionally associated with decreased AMP kinase activity."J Cell Physiol. 221(3):740-749. doi: 10.1002/jcp.21917.

16. Kim JH, Lee DE, Choi SH, Cha JH, Bak EJ, Yoo YJ. (2014) "Diabetic characteristics and alveolar bone loss in streptozotocin- and streptozotocin-nicotinamide-treated rats with periodontitis". J Periodontal Res. 49(6):792800. doi: $10.1111 /$ jre. 12165

17. Kostenuik PJ, Shalhoub V. (2001) "Osteoprotegerin: a physiological and pharmacological inhibitor of bone resorption." Curr Pharm Des. 7(8):613-635.

18. Lane NE, Yao W, Balooch M, Nalla RK, Balooch G, Habelitz S, Kinney JH, Bonewald LF. (2006) "Glucocorticoid-Treated mice have localized changes in trabecular bone material properties and osteocyte lacunar size that are not observed in Placebo-treated or estrogen deficient mice." J. Bone. Miner. Res., 21: 466476. doi:10.1359/JBMR.051103

19. Lecka-Czernik B, Moerman EJ, Grant DF, Lehmann JM, Manolagas SC, Jilka RL (2002) "Divergent effects of selective peroxisome proliferator-activated receptor-gamma 2 ligands on adipocyte versus osteoblast differentiation." Endocrinology. 143(6):2376-2384. doi:10.1210/endo.143.6.8834

20. Leidig-Bruckner G, Grobholz S, Bruckner T, Scheidt-Nave C, Nawroth P, Schneider JG. (2014) "Prevalence and determinants of osteoporosis in patients with type 1 and type 2 diabetes mellitus". Endocr Disord. 11;14:33. doi: 10.1186/1472-6823-14-33.

21. Madsen A, Bozickovic O, Bjune JI, Mellgren G, Sagen JV (2015) "Metformin inhibits hepatocellular glucose, lipid and cholesterol biosynthetic pathways by transcriptionally suppressing steroid receptor coactivator 2 (SRC-2)." Sci Rep.9; 5:16430. doi: 10.1038/srep16430

22. Mai QG, Zhang ZM, Xu S, Lu M, Zhou RP, Zhao L, Jia CH, Wen ZH, Jin DD, Bai XC. (2011) "Metformin stimulates osteoprotegerin and reduces RANKL expression in osteoblasts and ovariectomized rats". J Cell Biochem.. 112 (10):2902-2909. doi: 10.1002/jcb.23206.

23. Marycz K, Tomaszewski KA, Kornicka K, Henry BM, Wroński S, Tarasiuk J, Maredziak M (2016) "Metformin Decreases Reactive Oxygen Species, Enhances Osteogenic Properties of Adipose-Derived Multipotent Mesenchymal Stem Cells In Vitro, and Increases Bone Density In Vivo.” Oxid Med Cell Longev. 97858-90. doi: $10.1155 / 2016 / 9785890$

24. Masiello P, Broca C, Gross R, Roye M, Manteghetti M, Hillaire-Buys D, Novelli M, Ribes G. (1998) "Experimental NIDDM: Development of a New Model in Adult Rats Administered Streptozotocin and Nicotinamide".Diabetes. 47(2): 224-229.

25. McCarthy AD, Cortizo AM, Sedlinsky CWorld (2016) "Metformin revisited: Does this regulator of AMPactivated protein kinase secondarily affect bone metabolism and prevent diabetic osteopathy". J. Diabetes. 25; 7(6):122-33. doi: 10.4239/wjd.v7.i6.122.

26. Nathan DM, Buse JB, Davidson MB, Heine RJ, Holman RR, Sherwin R, Zinman B (2006) "Management of hyperglycemia in type 2 diabetes: A consensus algorithm for the initiation and adjustment of therapy: a consensus statement from the American Diabetes Association and the European Association for the Study of Diabetes." Diabetes Care; 29(8):1963. doi:10.2337/dc06-9912

27. Nicodemus KK, and Folsom AR. (2001) "Type 1 and type 2 diabetes and incident hip fractures in postmenopausal women". Diabetes care. 24:1192-7. 
28. Okonkwo P.O. and Okoye ZSC (2014) "Comparative Effects of Antidiabetic Drug, Metformin and Deferoxamine, on Serum Lipids, Serum Ferritin and Endocrine Indicators of Diabetes Mellitus Complications in Sreptozotocin Diabetic Rats" International Journal of Biochemistry Research \& Review. 4(6): 536-549,

29. Rojas LB, Gomes MB (2013) "Metformin: an old but still the best treatment for type 2 diabetes." Diabetology \& Metabolic Syndrome, 15; 5 (1):6. doi: 10.1186/1758-5996-5-6.

30. Rubin MR. (2015) "Bone cells and bone turnover in diabetes mellitus". Curr Osteoporos Rep. 13(3):186-191. doi:10.1007/s11914-015-0265-0

31. Schwartz AV (2017) "Review Diabetes, bone and glucose-lowering agents: clinical outcomes." Diabetologia. Jul; 60(7):1170-1179. doi: 10.1007/s00125-017-4283-6.

32. Szkudelski T, Zywert A, Szkudelska K. (2013) "Metabolic disturbances and defects in insulin secretion in rats with streptozotocin-nicotinamide-induced diabetes." Res. Physiol.; 62(6):663-670.

33. Szkudelski T. (2012) "Streptozotocin-nicotinamide-induced diabetes in the rat. Characteristics of the experimental model.” Exp Biol Med (Maywood).237(5):481-490. doi: 10.1258/ebm.2012.011372

34. Udagawa N, Takahashi N, Yasuda H, Mizuno A, Itoh K, Ueno Y, Shinki T, Gillespie MT, Martin TJ, Higashio K, Suda T.(2000) "Osteoprotegerin produced by osteoblasts is an important regulator in osteoclast development and function.” Endocrinology. 141(9):3478-3484. Doi :10.1210/endo.141.9.7634

35. Vestergaard P, Rejenmark L, Mosekilde L.(2005) "Relative fracture risk in patients with diabetes mellitus, and the impact of insulin and oral antidiabetic medication on relative fracture risk". Diabetologia. 48: 1292-9. doi:10.1007/s00125-005-1786-3

36. Vianna A. G. D., Sanches C. P. and Barreto F. C (2017) "Effects of type 2 diabetes therapies on bone metabolism. Diabetol Metab Syndr. 9: 75. doi: 10.1186/s13098-017-0274-5

37. Viviana Lacava, Vincenzo Pellicanò, Carmen Ferrajolo, Valeria Cernaro, Luca Visconti, Giovanni Conti (2017) "Novel avenues for treating diabetic nephropathy: new investigational drugs." Journal of Expert Opinion on Investigational Drugs 26:445-462.doi: 10.1080/13543784.2017.1293039.

38. Xue Han, Yu-Long Tao, Ya-Ping Deng, Jia-Wen Yu , Jian Cai, Guo-Fei Ren and Yuan-Nan Sun, Guo-Jun Jiang (2017) "Metformin ameliorates insulitis in STZ induced diabetic mice". Peer J. doi: 10.7717/peerj.3155.

39. Zhong Q, Itokawa T, Sridhar S, Ding KH, Xie D, Kang B, Bollag WB, Bollag RJ, Hamrick M, Insogna K, Isales CM. (2007) "Effects of glucose-dependent insulinotropic peptide on osteoclast function." Am J Physiol Endocrinol Metab. 292 (2), 543-548. doi:10.1152/ajpendo.00364.2006. 